\title{
Multivariate Connectome-Based Symptom Mapping in Post- Stroke Patients: Networks Supporting Language and Speech
}

\author{
Grigori Yourganov, ${ }^{1 \star}$ Julius Fridriksson, ${ }^{2}$ Chris Rorden, ${ }^{3}$ Ezequiel Gleichgerrcht, ${ }^{1}$ and Leonardo Bonilha ${ }^{1}$ \\ ${ }^{1}$ Department of Neurology, Medical University of South Carolina, Charleston, South Carolina 29425, and Departments of ${ }^{2}$ Communication Sciences and \\ Disorders and ${ }^{3}$ Psychology, University of South Carolina, Columbia, South Carolina 29208
}

\begin{abstract}
Language processing relies on a widespread network of brain regions. Univariate post-stroke lesion-behavior mapping is a particularly potent method to study brain-language relationships. However, it is a concern that this method may overlook structural disconnections to seemingly spared regions and may fail to adjudicate between regions that subserve different processes but share the same vascular perfusion bed. For these reasons, more refined structural brain mapping techniques may improve the accuracy of detecting brain networks supporting language. In this study, we applied a predictive multivariate framework to investigate the relationship between language deficits in human participants with chronic aphasia and the topological distribution of structural brain damage, defined as post-stroke necrosis or cortical disconnection. We analyzed lesion maps as well as structural connectome measures of whole-brain neural network integrity to predict clinically applicable language scores from the Western Aphasia Battery (WAB). Out-of-sample prediction accuracy was comparable for both types of analyses, which revealed spatially distinct, albeit overlapping, networks of cortical regions implicated in specific aspects of speech functioning. Importantly, all WAB scores could be predicted at better-than-chance level from the connections between gray-matter regions spared by the lesion. Connectome-based analysis highlighted the role of connectivity of the temporoparietal junction as a multimodal area crucial for language tasks. Our results support that connectome-based approaches are an important complement to necrotic lesion-based approaches and should be used in combination with lesion mapping to fully elucidate whether structurally damaged or structurally disconnected regions relate to aphasic impairment and its recovery.
\end{abstract}

Key words: aphasia; brain lesions; connectome; multivariate analysis; stroke; support vector regression

\section{Significance Statement}

We present a novel multivariate approach of predicting post-stroke impairment of speech and language from the integrity of the connectome. We compare it with multivariate prediction of speech and language scores from lesion maps, using cross-validation framework and a large $(n=90)$ database of behavioral and neuroimaging data from individuals with post-stroke aphasia. Connectome-based analysis was similar to lesion-based analysis in terms of predictive accuracy and provided additional details about the importance of specific connections (in particular, between parietal and posterior temporal areas) for preserving speech functions. Our results suggest that multivariate predictive analysis of the connectome is a useful complement to multivariate lesion analysis, being less dependent on the spatial constraints imposed by underlying vasculature.

\section{Introduction}

A modern approach to neuroscience suggests the understanding of brain function as a consequence of signal processing within neuronal networks (Yuste, 2015). More fundamental functions,

\footnotetext{
Received Dec. 8, 2015; revised May 2, 2016; accepted May 5, 2016.

Author contributions: J.F., C.R., and L.B. designed research; G.Y. and J.F. performed research; G.Y., C.R., E.G., and L.B. contributed unpublished reagents/analytic tools; G.Y. analyzed data; G.Y., J.F., E.G., and L.B. wrote the paper. This research was supported by National Institute on Deafness and Other Communication Disorders Grant DC014021 (L.B.) and National Institutes of Health Grant DC009571 (J.F. and C.R.).

The authors declare no competing financial interests.

Correspondence should be addressed to Grigori Yourganov, Department of Neurology, Medical University of South Carolina, 96 Jonathan Lucas Street, CSB 301, Charleston, SC 29425. E-mail: yourgano@musc.edu.

DOI:10.1523/JNEUROSCI.4396-15.2016

Copyright $\odot 2016$ the authors $\quad 0270-6474 / 16 / 366668-12 \$ 15.00 / 0$
}

such as motor and sensory processing, are localized to relatively specific brain regions, whereas more complex higher-level functions are distributed over multimodal cortical networks (Mesulam, 1990; McIntosh, 1999; Sporns, 2014). This hierarchical organization allows for integration of information across specialized brain regions, providing the neuronal basis for supporting a wide repertoire of flexible and complex cognitive behaviors (Tononi, 2004; Bressler and Menon, 2010; Park and Friston, 2013). Notably, the theory of brain organization as a hierarchy of coordinated networks was developed by Alexander Luria, who relied on data from lesion studies (Luria, 1966); the concept was subsequently supported by many neuroimaging studies (Zeki et al., 1991; McIntosh, 2000; Hagmann et al., 2008; Bressler and Menon, 2010; Sporns, 2013). This organization sug- 
gests that brain-behavior relationships could be better elucidated by applying multivariate, rather than univariate, analysis (Schmah et al., 2010; as supported by several fMRI studies: Misaki et al., 2010; Yourganov et al., 2014). Lesion studies could also benefit from a multivariate approach (Smith et al., 2013; Mah et al., 2014; Yourganov et al., 2015; Zhang et al., 2014); instead of analyzing the effect of lesions in different locations independently, as is done in voxelwise lesion symptom mapping (VLSM; Bates et al., 2003), a multivariate method accounts for the interactions between spatial locations.

Using lesion data to infer the functions of specific brain regions has certain limitations for identifying critical neuroanatomy supporting higher cognitive functions. First, lesions disrupting only a few nodes of the underlying network can lead to functional interruption; as such, lesion-based analysis might identify the lesioned nodes as crucial for this interruption, but the spared nodes are likely to be missed. Second, lesion studies are typically performed in participants who are stroke survivors, in whom the anatomical distribution of damage relies on constraints imposed by cerebrovascular anatomy. Vascular lesions typically involve multiple structures within the same vascular perfusion bed, and this frequent co-occurrence of damaged regions prevents the statistical discrimination of the essential structures.

Recently, our group confirmed that cortical disconnection, i.e., the loss of white-matter fibers supporting a cortical region apparently intact after the stroke, contributes to the severity of language impairment (Bonilha et al., 2014). In fact, cortical disconnections can lead to deficits that are as severe as those caused by cortical necrosis (Fridriksson et al., 2007). Importantly, poststroke cortical deafferentation can be pervasive and invisible to conventional structural MRI (Bonilha et al., 2014), such as T1- or T2-weighted images. Therefore, behaviorally relevant poststroke brain damage can be understood as a combination of cortical necrosis and cortical disconnection. The structural human brain connectome now provides an unprecedented tool to comprehensively assess white-matter integrity after stroke. The structural connectome (Hagmann et al., 2008) is a recent development relying on magnetic resonance imaging to assess gray-matter anatomy and axonal connectivity inferred from water diffusion within white-matter tracts. Connections between an individual's brain regions can be represented as a vector and analyzed with univariate or multivariate methods similarly to lesion maps.

In this study, we aimed to evaluate the cortical regions and neural networks supporting processing of basic clinical tasks assessing speech and language processing. For this purpose, we applied support vector regression (SVR; Smola and Schölkopf, 2004) to model the relationship between brain damage (defined by lesions or integrity of the connectome) and the severity of behavioral impairment. The accuracy of this model was evaluated by leave-one-participant-out cross-validation. We investigated the spatial overlap of the cortical networks revealed by connectome-based and lesion-based analyses. Moreover, we hypothesized that connectome-based mapping could disclose the importance of regions with less prevalence of post-necrotic lesion, i.e., more likely to be disconnected rather than directly damaged by the stroke.

\section{Materials and Methods}

\section{Participants}

Participants were recruited from the local community, and the study was approved by the Institutional Review Board at the University of South Carolina. Only individuals with aphasia resulting from a single ischemic or hemorrhagic stroke to the left hemisphere were included. Participants with lacunar infarcts or with damage that only involved the brainstem or cerebellum were excluded. The behavioral assessment of the participants took place between May 2007 and October 2014, and 90 individuals were included in the final data analyses. The mean \pm SD sample age was $58.8 \pm 12.1$ years (range, $31-81$ years), and 34 were women. All participants were at least 6 months after stroke, and the mean \pm SD time since stroke onset was $42.8 \pm 50$ months (range, 6-276 months).

\section{Behavioral evaluation}

Aphasic impairment was assessed using the Western Aphasia Battery (WAB; Kertesz, 1982). Of the 90 participants whose data constituted the final study sample, the following aphasia types were observed: (1) anomic (26 participants); (2) Broca's (30 participants); (3) conduction (nine participants); (4) global (eight participants); (5) Wernicke's (five participants); and (6) no aphasia (12 participants). In our study, we focused on four WAB scores that evaluate specific domains of speech and language (speech fluency, auditory comprehension, speech repetition, and oral naming) and on the summary score of overall aphasia severity, termed aphasia quotient (AQ).

\section{Imaging data}

MRI scanning was performed within $2 \mathrm{~d}$ of behavioral testing of language abilities. Images were acquired on a Siemens Trio 3T scanner equipped with a 12-element head coil located at the University of South Carolina. Three images were collected for each patient.

The first was a T1-weighted image using an MP-RAGE sequence with $1 \mathrm{~mm}$ isotropic voxels, a $256 \times 256$ matrix size, and a $9^{\circ}$ flip angle. For the first 25 individuals, we used a 160 slice sequence with TR of $2250 \mathrm{~ms}$, TI of $900 \mathrm{~ms}$, and TE of $4.52 \mathrm{~ms}$. For the latter 65 individuals, we used a 192 slice sequence with TR of $2250 \mathrm{~ms}$, TI of $925 \mathrm{~ms}$, and TE of 4.15 with parallel imaging [generalized autocalibrating partially parallel acquisitions (GRAPPA) of 2; 80 reference lines]. Each of these scans required $\sim 7$ min to acquire.

The second was a T2-weighted image using a sampling perfection with application optimized contrasts using a different flip angle evolution [3D-SPACE (sampling perfection with application optimized contrasts using different flip angle evolution)] sequence. This 3D TSE (Turbo Spin Echo) scan uses a TR of $2800 \mathrm{~ms}$, TE of $402 \mathrm{~ms}$, variable flip angle, and $256 \times 256$ matrix scan with 192 slices ( $1 \mathrm{~mm}$ thick), using parallel imaging (GRAPPA of 2; 120 reference lines).

The third was a diffusion EPI scan that uses 30 directions with $b=$ $1000 \mathrm{~s} / \mathrm{mm}^{2}$ and $b=2000 \mathrm{~s} / \mathrm{mm}^{2}$, TR of $6100 \mathrm{~ms}$, TE of $101 \mathrm{~ms}, 82 \times 82$ matrix, $222 \times 222 \mathrm{~mm}$ FOV, with parallel imaging, GRAPPA of $2(80$ reference lines), 45 contiguous $2.7 \mathrm{~mm}$ axial slices, and acquisition time of $390 \mathrm{~s}$.

Post-stroke gray-matter lesions. Lesions were manually drawn on the T2-weighted image by a neurologist (L.B.), who was blinded to the participant's language scores at the time of the lesion drawing. The T2 image was coregistered to the T1 image, and these parameters were used to reslice the lesion into the native T1 space. The resliced lesion maps were smoothed with a $3 \mathrm{~mm}$ full-width half-maximum Gaussian kernel to remove jagged edges associated with manual drawing. We then performed enantiomorphic normalization (Nachev et al., 2008) using SPM12 and MATLAB scripts that we developed as follows. First, a mirrored image of the T1 scan (reflected around the midline) was created, and this mirrored image was coregistered to the native T1 image. We then created a chimeric image based on the native T1 scan with the lesioned tissue replaced by tissue from the mirrored scan (using the smoothed lesion map to modulate this blending, feathering the lesion edge). The unified segmentation normalization of SPM12 (Ashburner and Friston, 2005) was used to warp this chimeric image to standard space, with the resulting spatial transform applied to the actual T1 scan and the lesion map. The normalized lesion map was then binarized, using a 50\% probability threshold. Figure 1 shows the overlap of lesions for our sample of participants.

Brain parcellation. To reduce the dimensionality of our data, we used a brain atlas developed by Faria et al. (2012) to divide the gray matter into 118 regions. We used two complimentary ways to characterize brain 
damage: (1) based on lesions; and (2) based on the connectome. To compute lesion-based damage, we aligned the anatomical brain atlas containing the parcellation with each individual's T1-weighted images. The T1-weighted images were segmented into probabilistic grayand white-matter maps, and the gray-matter map was divided into regions according to the atlas. Then, lesion-based damage was computed as the proportion of intact (i.e., not lesioned) voxels per each gray-matter region. Connectome-based damage was computed as the number of diffusion tensor imaging (DTI) tracts that connected each pair of the graymatter regions, as described in detail in the next section. For both types of the analysis, we excluded subcortical and cerebellar regions.

Structural brain connectome. To align the diffusion image with the lesion map, the T2weighted image (coregistered into the native T1-weighted image, thus corresponding to the native $\mathrm{T} 1$ space) was linearly normalized into the nondiffusion image from the diffusion MRI sequence (the B0 image) using FSL (FMRIB Software Library) FMRIB (Functional MRI of the Brain) Linear Image Registration Tool (affine registration with 12 parameters, correlation ration cost function and nearest neighbor interpolation). The resulting spatial transform was used to register the probabilistic maps of white and gray matter in native T1 space and the stroke lesion into the diffusion MRI space. Because the probabilistic gray-matter map was parcellated into regions of interest (ROIs) as described above (see Brain parcellation), this registration step yielded the spatial normalization of the atlas ROIs to diffusion space.

All subsequent connectome preprocessing steps were performed in diffusion space. Probabilistic tractography was applied to evaluate pairwise gray-matter structural connectivity. Tractography was estimated through the probabilistic method of FDT (FMRIB Diffusion Toolbox; Behrens et al., 2007) with FDT BEDPOST being used to build default distributions of diffusion parameters at each voxel, followed by probabilistic tractography using FDT probtrackX (parameters: 5000 individual pathways drawn through the probability distributions on principle fiber direction, curvature threshold set at 0.2, 200 maximum steps, step length of $0.5 \mathrm{~mm}$, and distance correction). The probabilistic white-matter map excluding the stroke lesion was used as the waypoint mask. As explained above, the probabilistic white-matter map was obtained from T1weighted images and spatially normalized into diffusion space. The connectivity between ROIs was defined as the number of streamlines arriving in one region when another ROI was seeded and vice versa. Specifically, the following process was iteratively performed for all ROIs: the seed space was defined as ROI $i$, with the probabilistic white-matter map set as the waypoint mask. This process resulted in one three-dimensional image in diffusion space, in which each voxel represented the number of connectivity streamlines of ROI $i$. The probabilistic gray-matter map divided into ROIs was then overlaid onto this image, and the number of connectivity streamlines was counted for every other ROI besides $i$. Thus, the weighted connectivity between the $i$ th and $j$ th regions was defined as the number of probabilistic streamlines arriving at $j$ th region when the $i$ th region was seeded, averaged with the number of probabilistic streamlines arriving at the $i$ th region when the $j$ th region was seeded. The calculation of the probabilistic streamlines was corrected based on the distance traveled by the streamline connecting $i$ th and $j$ th regions ("distance correction" built into probtrackX). To compensate for the unequal size of gray-matter regions, the number of streamlines connecting each pair of regions was divided by the sum of the volume of areas of these regions. Because we did not track the fibers within the lesion site, the entries of the connectome matrices that corresponded to the connections stemming from completely lesioned brain regions were set to zero (rather than being treated as missing data).
Table 1. Gray-matter regions used in lesion-based analysis

\begin{tabular}{ll}
\hline Superior frontal gyrus (posterior segment) & Inferior temporal gyrus \\
Superior frontal gyrus (prefrontal cortex) & Parahippocampal gyrus \\
Superior frontal gyrus (frontal pole) & Entorhinal area \\
Middle frontal gyrus (posterior segment) & Fusiform gyrus \\
Middle frontal gyrus (dorsal prefrontal cortex) & Superior occipital gyrus \\
Inferior frontal gyrus pars opercularis & Middle occipital gyrus \\
Inferior frontal gyrus pars orbitalis & Inferior occipital gyrus \\
Inferior frontal gyrus pars triangularis & Cuneus \\
Lateral fronto-orbital gyrus & Lingual gyrus \\
Middle fronto-orbital gyrus & Rostral anterior cingulate gyrus \\
Gyrus rectus & Dorsal anterior cingulate gyrus \\
Postcentral gyrus & Posterior cingulate gyrus \\
Precentral gyrus & Insula \\
Superior parietal gyrus & Amygdala \\
Supramarginal gyrus & Hippocampus \\
Angular gyrus & Cingulum (cingulate gyrus) \\
Precuneus & Cingulum (hippocampus) \\
Superior temporal gyrus & Posterior insula \\
Pole of superior temporal gyrus & Posterior superior temporal gyrus \\
Middle temporal gyrus & Posterior middle temporal gyrus \\
Pole of middle temporal gyrus & Posterior inferior temporal gyrus \\
\hline
\end{tabular}

\section{SVR}

To model the relationship between the brain damage and speech impairment, we used SVR with the linear kernel. The estimated linear model is

$$
y=w_{1} x_{1}+w_{2} x_{2}+\ldots+w_{N} x_{N}+c .
$$

Here, $y$ is the WAB subscore, each $x_{i}$ is a feature, which is either the proportion of intact voxels in the ith gray-matter region (for lesion-based symptom mapping) or the number of streamlines in the $i$ th link of the connectome (for connectome-based symptom mapping), $N$ is the number of features, and $c$ is the constant offset term.

For both lesion-based and connectome-based analyses, we preselected a set of features to exclude those that were considered too noisy or uninformative. In the first case, we only considered gray-matter regions that were damaged in at least 4 of 90 participants; there were 42 such regions (Table 1). In the second case, we only considered the left-hemisphere tracts whose right-hemisphere homologs were reproducible across participants. We subsequently ordered these links based on their weighted dispersion across subjects, selecting the highest reproducible $10 \%$. This process yielded 84 connections for additional analysis. These connections are displayed on Figure 2; the names of the regions that are connected by the links are provided in Table 2 .

The accuracy of the model was evaluated using a leave-oneparticipant-out cross-validation procedure. One participant was set 


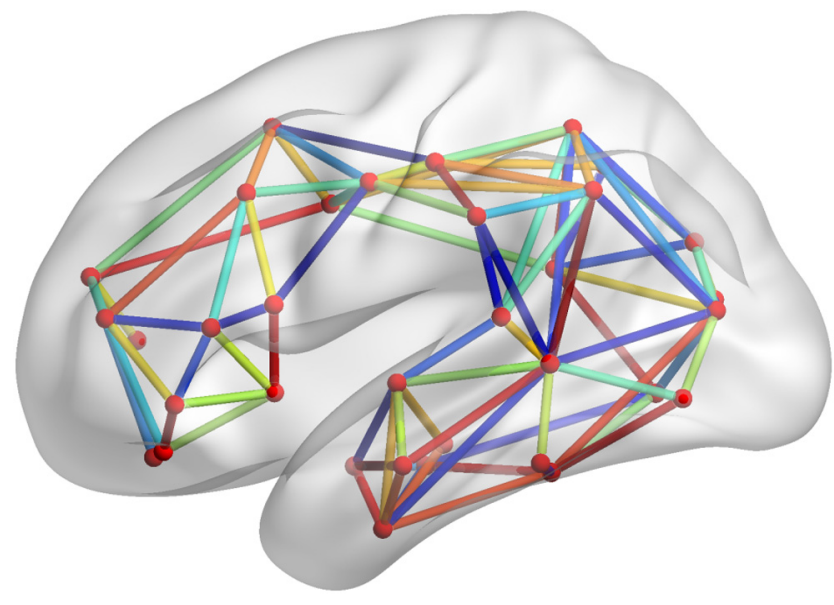

Figure 2. Left-hemisphere white-matter links that were used in connectome-based analysis. These links were selected based on the high reproducibility of their right-hemisphere homologs.

aside, and the brain damage from the remaining 89 participants was used to train the model (i.e., to estimate the coefficients $w_{i}$ and the offset $c$ in Eq. 1). Then, the model was tested by computing the WAB score for the left-out patient. The procedure was iterated so all of the 90 participants were, in turn, left out; this was repeated for two types of brain damage (lesion- and connectome-based) and for five WAB scores. Detailed descriptions of training and testing are given below.

Training the SVR model. During training, the set of 89 participants was used to compute the parameters of the linear model given in Equation 1, that is, the weights for each feature and the offset term. The first step was to scale the features to the $[0 \ldots 1]$ range, by dividing them by the global maximum value of all features in the training set. The next step was feature selection: we selected the features in which the damage was correlated (across the 89 participants) with the particular WAB score that we were estimating. The threshold for significance of correlation was 0.05 with Bonferroni's correction for multiple comparisons; the features that passed the threshold were retained for additional analysis.

The next step was the actual estimation of the model parameters; this was done using the LIBSVM (Library for Support Vector Machines) MATLAB library (Chang and Lin, 2011), which computes the weights $w_{i}$ and the offset $c$ (see Eq. 1) using the scores and damage data from the training set of participants. We performed two additional steps to improve the predictive performance of SVR. First, we ensured that the range of the SVM output was not too compressed. To ensure that the range of predicted WAB scores matched the range of the observed scores, we predicted the WAB scores of our training set of participants and compared the ranges of the predicted and observed training scores. We assumed that the relationship between the observed score $y_{i}$ and the predicted score $\hat{y}_{i}$ was linear:

$$
y_{1}=a \hat{y}_{i}+b
$$

The values of $a$ and $b$ were selected so that the line passed through the points $\left(\hat{y}_{\min }, y_{\min }\right)$ and $\left(\hat{y}_{\max }, y_{\max }\right)$.

After determining $a$ and $b$, we inspected the feature weights to ensure that they were positive. Our features measured the intactness of the gray or white matter; therefore, we had an a priori hypothesis that the feature weights would be non-negative, that is, if a value of any isolated feature is increased, we expect the WAB score to increase rather to decrease (higher $\mathrm{WAB}$ scores correspond to better preservation of function). Any negative feature weight was likely a product of overfitting (an attempt to model the noise that was specific to our training set); all such negative weights were changed to zeros.

Testing the SVR model. During testing, we scaled the features of the left-out patient (that is, divided them by the maximum value of the training set features), selected the features that were determined as predictive during training, and then, using Equation 1, computed the WAB score for the left-out patient. The output was then rescaled using the coefficients $a$ and $b$ according to Equation 2; note that these coefficients were computed on the training set without using any information about the left-out patient, therefore satisfying the requirement that no information from the test set could be used during training. The predicted WAB score was then clipped to the $[0 \ldots 10]$ range. When we were predicting speech fluency scores, the predicted score was also rounded to a nearest integer.

By iterating through all 90 participants, we obtained a set of predicted WAB scores. The accuracy of our prediction was evaluated by computing Pearson's correlation coefficient between the actual and predicted WAB scores.

Visualization of feature weights. The feature weights in the linear SVR model (specified as $w_{1}, w_{2}, \ldots, w_{N}$ in Eq. 1) characterize the importance of each feature, relative to other features, for predicting the score. For visualization, we trained the linear SVR model on all 90 available participants to achieve as complete representation of our dataset as possible. The feature weights were then divided by the SD of feature weights and projected onto a standard brain template using BrainNet software (Xia et al., 2013).

\section{Prediction from lesion size}

In addition to lesion-based and connectome-based predictions, we predicted the behavioral scores from the size of the lesion (i.e., the number of voxels within the lesion map in spatially normalized brains). Prediction was performed using the leave-one-patient-out framework, with lesion size serving as the only predictor. Instead of using SVR (which requires more than one predictor), we used simple linear regression. At each iteration, the behavioral score for the left-out patient was estimated from the linear regression model that was computed using the remaining patients.

\section{Results}

The accuracy of predicting WAB scores from the lesion size was significantly better than chance, as demonstrated in Table 3. This table lists Pearson's correlation coefficient between actual and predicted WAB scores (fluency, auditory comprehension, repetition, naming, and AQ) and the associated $p$ value.

Because the lesion affects the gray matter and the connectome, the predictors in lesion-based analysis were correlated with the predictors in connectome-based analysis. The table of correlations is shown on Figure 3 (abbreviations are defined in Table 6; additional abbreviations used in Fig. 3: SFG, superior frontal gyrus; PFC, prefrontal cortex; MFOG, middle fronto-orbital gyrus; RG, gyrus rectus; SPG, superior parietal gyrus; $\mathrm{PcCu}$, precuneus; ENT, entorhinal area; FuG, fusiform gyrus; SOG/IOG, superior/inferior occipital gyrus; $\mathrm{Cu}$, cuneus; LG, lingual gyrus; ACC/PCC, anterior/posterior cingulate cortex; Hippo, hippocampus; CGC, cingulate gyrus; $\mathrm{CGH}$, hippocampal part of the cingulate gyrus). Tables 4 and 5 summarize the accuracy of predicting WAB scores from lesion data (Table 4) and from connectome data (Table 5). These tables report the accuracy of prediction and the number of features with nonzero weights (the remaining features had zero weights and therefore did not participate in predicting the score). The $p$ values are uncorrected for multiple comparisons; however, all correlations are significant ( $p<0.000005$ for each of the eight predictions). Figures $4-6$ display the corresponding scatter plots.

For both types of analysis (lesion- and connectome-based), prediction of speech fluency scores was the most accurate, perhaps because of the fact that the fluency scores were whole numbers whereas the other three scores were fractional. For three scores (fluency, repetition, and naming), prediction was more accurate for lesion-based analysis; auditory comprehension scores were more accurately predicted from the connectome. 
Table 2. White-matter connections used in connectome-based analysis

\begin{tabular}{|c|c|}
\hline Superior frontal gyrus (posterior segment) ↔ superior frontal gyrus (prefrontal cortex) & Superioroccipitalgyrus $\leftrightarrow$ cuneus \\
\hline Superior frontal gyrus (posterior segment) $\leftrightarrow$ middle frontal gyrus (posterior segment) & Middle occipital gyrus $\leftrightarrow$ cuneus \\
\hline Superior frontal gyrus (prefrontal cortex) $\leftrightarrow$ middle frontal gyrus (dorsal prefrontal cortex) & Parahippocampal gyrus $\leftrightarrow$ lingual gyrus \\
\hline Middle frontal gyrus (posterior segment) $\leftrightarrow$ middle frontal gyrus (dorsal prefrontal cortex) & Fusiform gyrus $\leftrightarrow$ lingual gyrus \\
\hline Middle frontal gyrus (posterior segment) $\leftrightarrow$ inferior frontal gyrus pars opercularis & Middle occipital gyrus $\leftrightarrow$ lingual gyrus \\
\hline Middle frontal gyrus (dorsal prefrontal cortex) $\leftrightarrow$ inferior frontal gyrus pars orbitralis & Superior frontal gyrus (prefrontal cortex) $\leftrightarrow$ rostral anterior cingulate gyrus \\
\hline Middle frontal gyrus (posterior segment) $\leftrightarrow$ inferior frontal gyrus pars triangularis & Superior frontal gyrus (posterior segment) $\leftrightarrow$ dorsal ant. cingulate gyrus \\
\hline Middle frontal gyrus (dorsal prefrontal cortex) ↔ inferior frontal gyrus pars triangularis & Superior frontal gyrus (prefrontal cortex) $\leftrightarrow$ dorsal anterior cingulate gyrus \\
\hline Inferior frontal gyrus pars opercularis $\leftrightarrow$ inferior frontal gyrus pars triangularis & Postcentral gyrus $\leftrightarrow$ dorsal anterior cingulate gyrus \\
\hline Inferior frontal gyrus pars orbitralis ↔ inferior frontal gyrus pars triangularis & Precentral gyrus $\leftrightarrow$ dorsal anterior cingulate gyrus \\
\hline Middle frontal gyrus (dorsal prefrontal cortex) $\leftrightarrow$ lateral fronto-orbital gyrus & Superior parietal gyrus $\leftrightarrow$ dorsal anterior cingulate gyrus \\
\hline Inferior frontal gyrus pars orbitralis $\leftrightarrow$ lateral fronto-orbital gyrus & Superior parietal gyrus $\leftrightarrow$ posterior cingulate gyrus \\
\hline Superior frontal gyrus (prefrontal cortex) $\leftrightarrow$ gyrus rectus & Precuneus $\leftrightarrow$ posterior cingulate gyrus \\
\hline Superior frontal gyrus (posterior segment) $\leftrightarrow$ postcentral gyrus & Superior occipital gyrus $\leftrightarrow$ posterior cingulate gyrus \\
\hline Superior frontal gyrus (posterior segment) $\leftrightarrow$ precentral gyrus & Middle occipital gyrus $\leftrightarrow$ posterior cingulate gyrus \\
\hline Middle frontal gyrus (posterior segment) $\leftrightarrow$ precentral gyrus & Lingual gyrus $\leftrightarrow$ posterior cingulate gyrus \\
\hline Inferior frontal gyrus pars opercularis $\leftrightarrow$ precentral gyrus & Dorsal anterior cingulate gyrus $\leftrightarrow$ posterior cingulate gyrus \\
\hline Postcentral gyrus $\leftrightarrow$ precentral gyrus & Inferior frontal gyrus pars opercularis $\leftrightarrow$ insula \\
\hline Postcentral gyrus $\leftrightarrow$ Superior parietal gyrus & Inferior frontal gyrus pars orbitralis $\leftrightarrow$ insula \\
\hline Precentral gyrus $\leftrightarrow$ Superior parietal gyrus & Inferior frontal gyrus pars triangularis $\leftrightarrow$ insula \\
\hline Postcentral gyrus $\leftrightarrow$ supramarginal gyrus & Lateral fronto-orbital gyrus $\leftrightarrow$ insula \\
\hline Precentral gyrus $\leftrightarrow$ supramarginal gyrus & Superior temporal gyrus $\leftrightarrow$ amygdala \\
\hline Postcentral gyrus $\leftrightarrow$ angular gyrus & Middle temporal gyrus $\leftrightarrow$ amygdala \\
\hline Precentral gyrus $\leftrightarrow$ angular gyrus & Inferior temporal gyrus $\leftrightarrow$ amygdala \\
\hline Superior parietal gyrus $\leftrightarrow$ angular gyrus & Parahippocampal gyrus $\leftrightarrow$ amygdala \\
\hline Supramarginal gyrus $\leftrightarrow$ angular gyrus & Superior temporal gyrus $\leftrightarrow$ hippocampus \\
\hline Postcentral gyrus $\leftrightarrow$ precuneus & Inferior temporal gyrus $\leftrightarrow$ hippocampus \\
\hline Superior parietal gyrus $\leftrightarrow$ precuneus & Superior parietal gyrus $\leftrightarrow$ posterior superior temporal gyrus \\
\hline Superior temporal gyrus $\leftrightarrow$ middle temporal gyrus & Supramarginal gyrus $\leftrightarrow$ posterior superior temporal gyrus \\
\hline Superior temporal gyrus $\leftrightarrow$ inferior temporal gyrus & Angular gyrus $\leftrightarrow$ posterior superior temporal gyrus \\
\hline Middle temporal gyrus $\leftrightarrow$ inferior temporal gyrus & Superior temporal gyrus $\leftrightarrow$ posterior superior temporal gyrus \\
\hline Inferior temporal gyrus $\leftrightarrow$ parahippocampal gyrus & Superior parietal gyrus $\leftrightarrow$ posterior middle temporal gyrus \\
\hline Inferior temporal gyrus $\leftrightarrow$ fusiform gyrus & Supramarginal gyrus $\leftrightarrow$ posterior middle temporal gyrus \\
\hline Parahippocampal gyrus $\leftrightarrow$ fusiform gyrus & Angular gyrus $\leftrightarrow$ posterior middle temporal gyrus \\
\hline Superior parietal gyrus $\leftrightarrow$ superior occipital gyrus & Superior temporal gyrus $\leftrightarrow$ posterior middle temporal gyrus \\
\hline Precuneus $\leftrightarrow$ superior occipital gyrus & Middle temporal gyrus $\leftrightarrow$ posterior middle temporal gyrus \\
\hline Superior parietal gyrus $\leftrightarrow$ middle occipital gyrus & Inferior temporal gyrus $\leftrightarrow$ posterior middle temporal gyrus \\
\hline Angular gyrus $\leftrightarrow$ middle occipital gyrus & Middle occipital gyrus $\leftrightarrow$ posterior middle temporal gyrus \\
\hline Fusiform gyrus $\leftrightarrow$ middle occipital gyrus & Inferior occipital gyrus $\leftrightarrow$ posterior middle temporal gyrus \\
\hline Superior occipital gyrus $\leftrightarrow$ middle occipital gyrus & Posterior superior temporal gyrus $\leftrightarrow$ posterior middle temporal gyrus \\
\hline Fusiform gyrus $\leftrightarrow$ inferior occipital gyrus & Fusiform gyrus $\leftrightarrow$ posterior inferior temporal gyrus \\
\hline Middle occipital gyrus $\leftrightarrow$ inferior occipital gyrus & Posterior middle temporal gyrus $\leftrightarrow$ posterior inferior temporal gyrus \\
\hline
\end{tabular}

Table 3. Accuracy of predicting WAB scores from lesion size

\begin{tabular}{ll}
\hline WAB score & Prediction accuracy \\
\hline Fluency & $0.6971(p=2.32427 \mathrm{e}-14)$ \\
Auditory comprehension & $0.5698(p=4.60602 \mathrm{e}-09)$ \\
Repetition & $0.5924(p=7.69624 \mathrm{e}-10)$ \\
Naming & $0.5712(p=4.11218 \mathrm{e}-09)$ \\
AQ & $0.6342(p=1.93589 \mathrm{e}-11)$ \\
\hline
\end{tabular}

However, when the correlation coefficients were transformed to $Z$ values using Fisher's $r$-to- $Z$ transform, the difference between lesion- and connectome-based predictions was not significant ( $p=0.072$ for the AQ score; $p>0.15$ for the remaining four scores). Lesion-based analysis tended to overestimate the WAB scores, e.g., the predicted scores were better than the actual scores. In contrast, connectome-based analysis tended to underestimate the scores. When Tables 3-5 are compared, we can see that lesion size is approximately as predictive as ROI-specific damage and connectome integrity (there was no significant difference in $r$-to- $Z$ transformed correlations; $p>0.15$ ); this is expected given the correlation between overall lesion size and amount of damage in some regions (Fig. 3).
The features that drove the prediction (see Materials and Methods, Visualization of feature weights) are visualized in Figures 7-9; only the features with loadings $>1$ are displayed. Table 6 lists the brain regions that correspond to the labels on these figures. For lesion-based analysis, the most important regions for predicting fluency scores were the precentral gyrus, opercular part of inferior frontal gyrus, and the posterior part of the superior temporal gyrus (PSTG); for predicting auditory comprehension scores, the PSTG, the triangular part of inferior frontal gyrus, the pole and the posterior part of the middle temporal gyrus, the amygdala, and the dorsal part of the middle frontal gyrus; for predicting speech repetition scores, the posterior part of the superior and the middle temporal gyri; and, finally, for predicting naming scores, the PSTG, the pole of the superior and middle temporal gyri, and the triangular part of the inferior frontal gyrus. For the connectome-based analysis, the most important connection for predicting speech fluency scores was between the precentral gyrus and pars opercularis; for predicting auditory comprehension scores, the connection between the insula and pars orbitalis; for predicting repetition scores, the connections between the angular gyrus and the PSTG and be- 


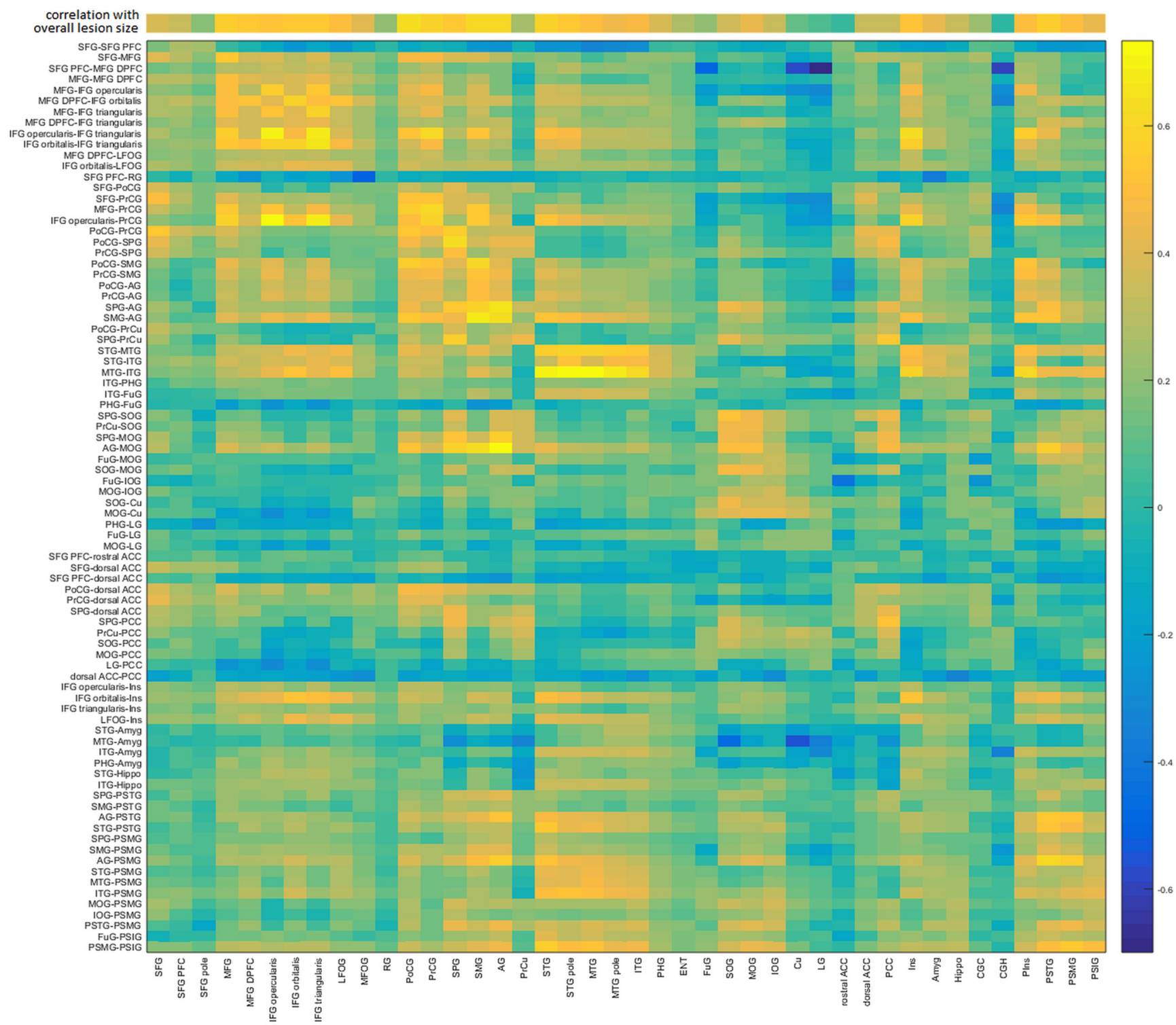

Figure 3. Correlation of features used in lesion-based and connectome-based analysis. The top row displays the correlation between the ROI damage and overall lesion size.

Table 4. Accuracy of predicting WAB scores from lesions

\begin{tabular}{llll}
\hline WAB score & $\begin{array}{l}\text { Correlation of } \\
\text { predicted and } \\
\text { actual scores }\end{array}$ & Pvalue & $\begin{array}{l}\text { Number of } \\
\text { predictive regions } \\
\text { (of 42) }\end{array}$ \\
\hline Speech fluency & 0.7054 & $8.32 \mathrm{e}-15$ & 20 \\
Auditory comprehension & 0.4560 & $6.28 \mathrm{e}-06$ & 16 \\
Speech repetition & 0.6189 & $7.92 \mathrm{e}-11$ & 18 \\
Naming & 0.5761 & $2.84 \mathrm{e}-09$ & 15 \\
$\mathrm{AQ}$ & 0.6936 & $3.5 \mathrm{e}-14$ & 22 \\
\hline
\end{tabular}

tween the angular and the middle occipital gyri; and, finally, for predicting naming scores, the links that connect the angular gyrus to the PSTG, the postcentral gyrus, and the PSTG, as well as the connection between the middle and the inferior temporal gyri.

There was an overall agreement between lesion-based and connectome-based analyses in identifying the parts of the brain relevant for speech modalities measured by WAB scores. For example, both types of analysis identified the PSTG and the connections stemming from it, to have an important contribution
Table 5. Accuracy of predicting WAB scores from connectomes

\begin{tabular}{llll}
\hline WAB score & $\begin{array}{l}\text { Correlation of } \\
\text { predicted and } \\
\text { actual scores }\end{array}$ & $P$ value & $\begin{array}{l}\text { Number of } \\
\text { predictive links } \\
\text { (of 84) }\end{array}$ \\
\hline Speech fluency & 0.6332 & $2.13 \mathrm{e}-11$ & 14 \\
Auditory comprehension & 0.5233 & $1.21 \mathrm{e}-07$ & 7 \\
Speech repetition & 0.5171 & $1.81 \mathrm{e}-07$ & 8 \\
Naming & 0.5341 & $5.90 \mathrm{e}-08$ & 9 \\
AQ & 0.5603 & $9.3 \mathrm{e}-09$ & 11 \\
\hline
\end{tabular}

for predicting all five WAB scores. Also, precentral gyrus and pars opercularis were identified as important contributors to fluent speech production by both lesion- and connectome-based analyses. As another example, the link between the angular gyrus and PSTG was highly relevant for prediction of repetition and naming scores; both areas are likewise relevant in lesion-based analysis. Conversely, some features were detected by one type of the analysis but not the other. One such example is the polar regions of the temporal lobe (which are important contributors in lesionbased prediction of auditory comprehension, repetition, and 
fluency $(r=0.71)$

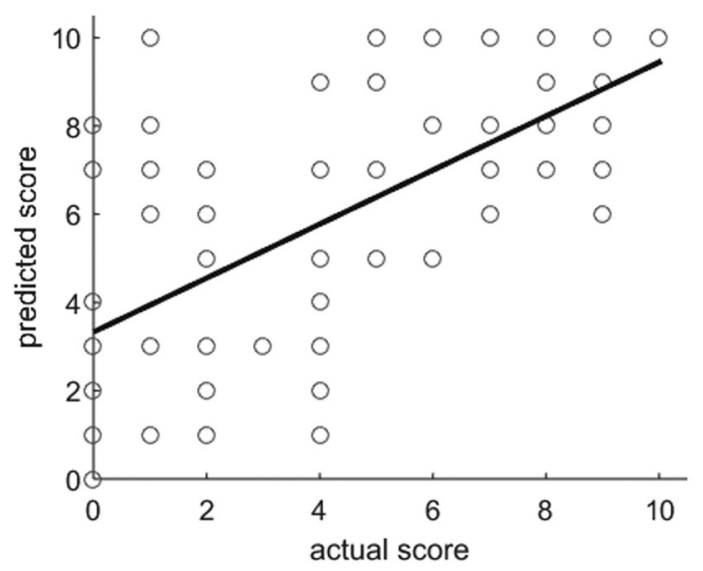

repetition $(r=0.62)$

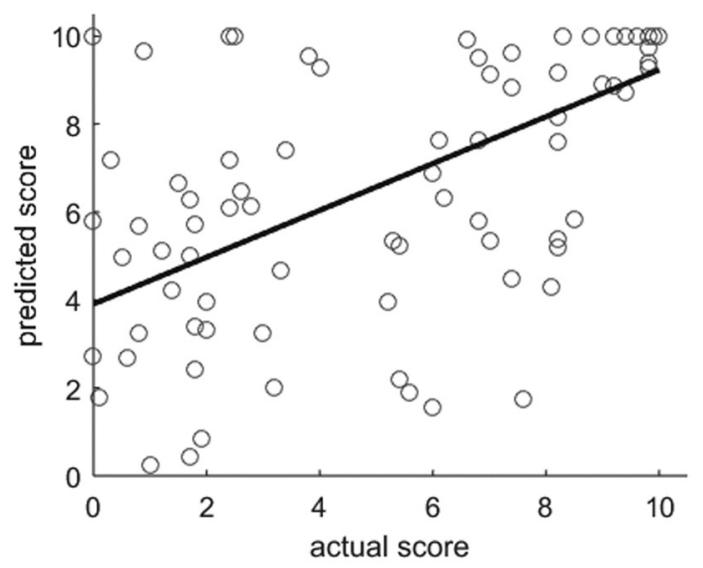

auditory comprehension $(r=0.46)$

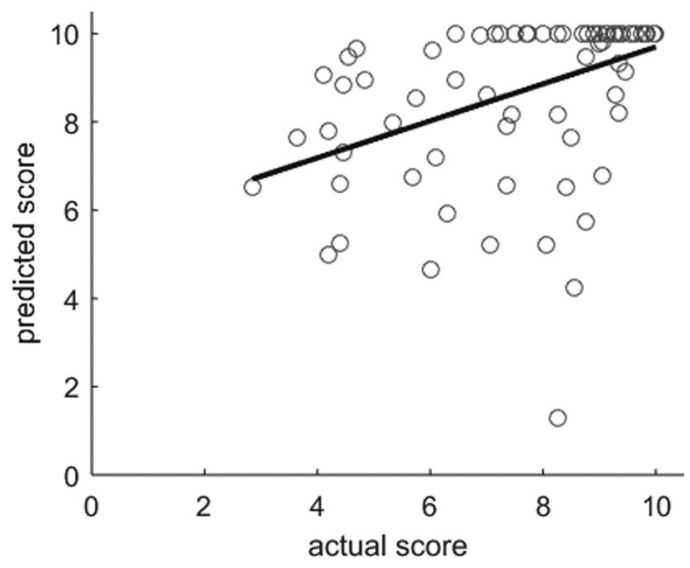

naming $(r=0.58)$

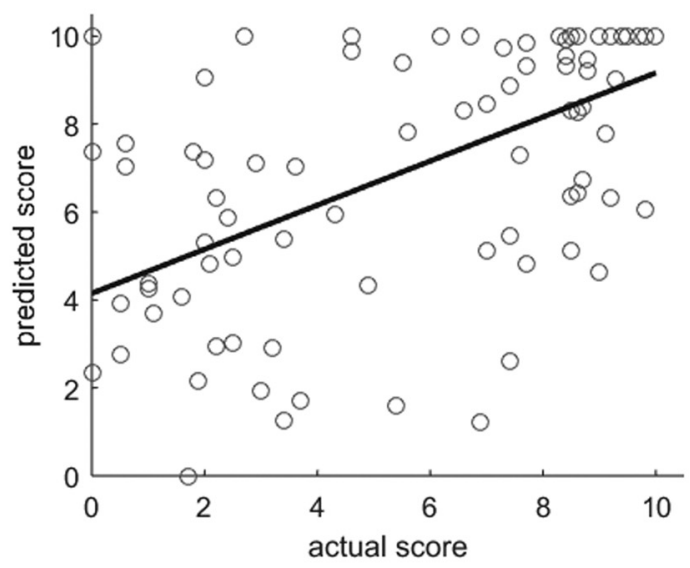

Figure 4. Scatter plots of actual versus predicted WAB scores (each dot representing a patient) for lesion-based analysis, with the corresponding linear regression line. Pearson's correlations coefficients between actual and predicted scores are specified above each plot.

naming scores). Moreover, the frontal regions were predictive of naming scores based on their lesions but not on their connectivity; the contribution of parietal regions to auditory comprehension prediction was detected by connectivity-based analysis but not by lesion-based analysis; the connection between pars orbitalis and the dorsal part of middle frontal gyrus was strongly predictive of speech repetition scores, but damage to those regions was not predictive.

Overall, our results demonstrate that lesion-based and connectome-based predictions are comparable in terms of accuracy but not altogether similar in terms of the spatial features that drive the predictions. To further evaluate the complementarity of these two analyses, we predicted the WAB scores from the combination of lesion-based and connectome-based features (i.e., regional damage and probabilistic fiber counts). Because the regional damage is measured on scale a from zero (no damage) to one (region completely destroyed by lesion), we scaled the connectome data to the same range, dividing all values by the largest connectome value across all subjects and connections. As shown in Table 7, the accuracy of predictions is similar to the results obtained from the lesion maps (Table 4) and from the connectomes (Table 5).

To further investigate the question of whether the connectome contained information not captured by lesion maps, we performed additional analyses: using the same leave-one-patient- out framework, we identified the gray-matter regions that were $>5 \%$ damaged in the left-out patient and removed the connections stemming from these regions from the training set. Therefore, the SVR model was trained on the connections between relatively intact cortical regions. Table 8 displays the resulting prediction accuracy, computed as Pearson's correlation coefficient between the actual and predicted WAB scores. For all WAB scores except for $\mathrm{AQ}$, the correlation between the actual and predicted WAB scores was significantly better than chance.

\section{Discussion}

Converging evidence from lesion and functional neuroimaging studies has demonstrated that the processes supporting speech production and comprehension recruit a widespread network of brain regions, although the spatial extent of such recruitment is a subject of ongoing debate (Dronkers et al., 2004; Hickok and Poeppel, 2007; Henseler et al., 2014). A stroke can disrupt the functioning of this network in several ways. The lesion might destroy a gray-matter region that serves as a node of the network; it can also damage a white-matter tract and therefore disrupt the communication between network nodes that have been spared by the lesion (Geschwind, 1965). In addition, a white-matter tract can be damaged by post-stroke Wallerian degeneration (Thomalla et al., 2005). This focal damage might affect the communication between the parts of the network that are located far from the 
fluency $(r=0.63)$

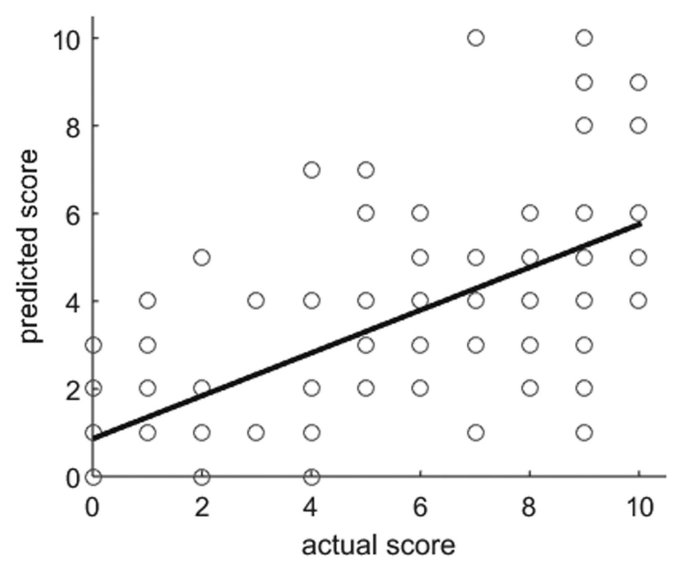

repetition $(r=0.52)$

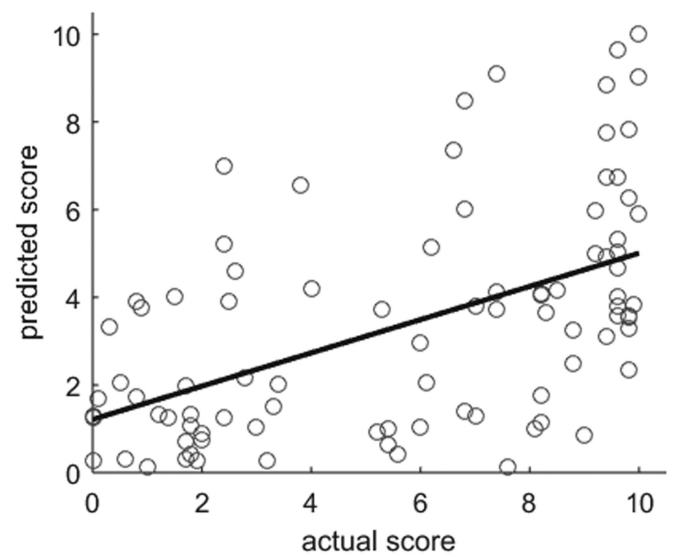

auditory comprehension $(r=0.52)$

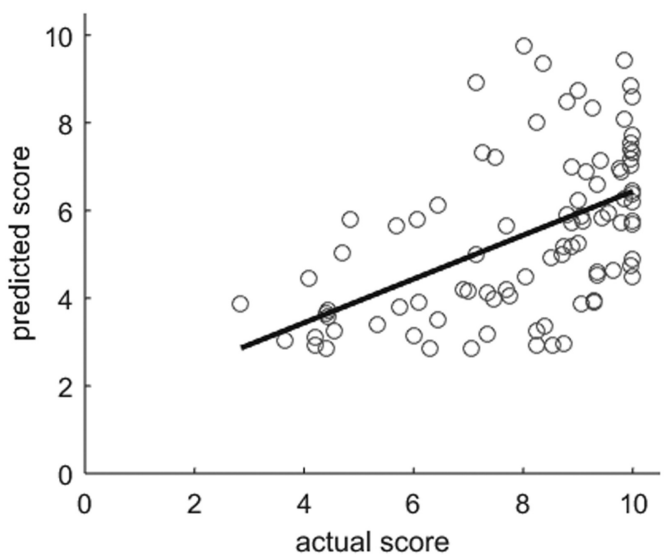

naming $(r=0.53)$

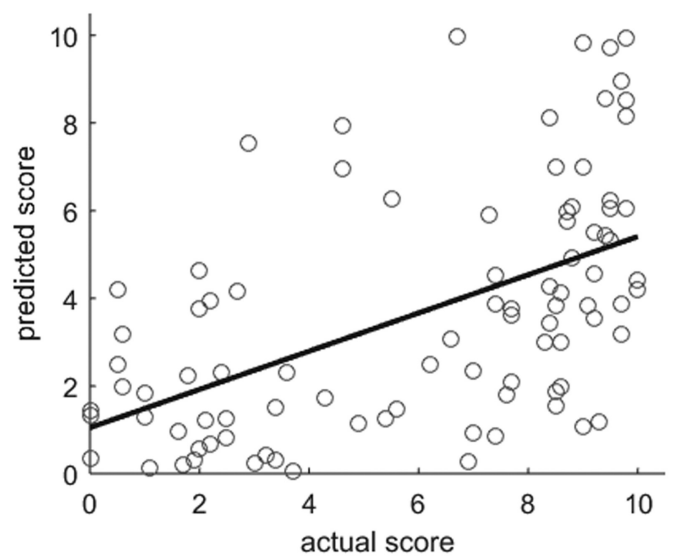

Figure 5. Scatter plots of actual versus predicted WAB scores (each dot representing a patient) for connectome-based analysis, with the corresponding linear regression line. Pearson's correlations coefficients between actual and predicted scores are specified above each plot.

lesion ( $r=0.69)$

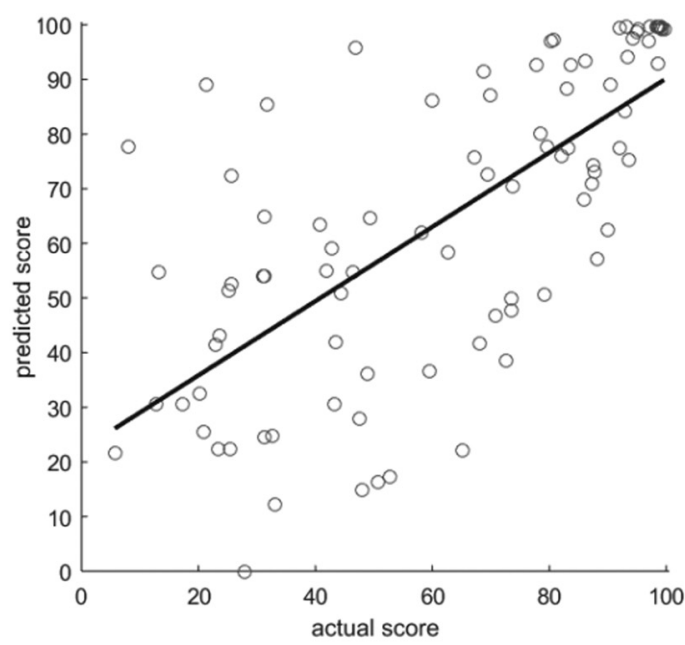

connectome $(r=0.56)$

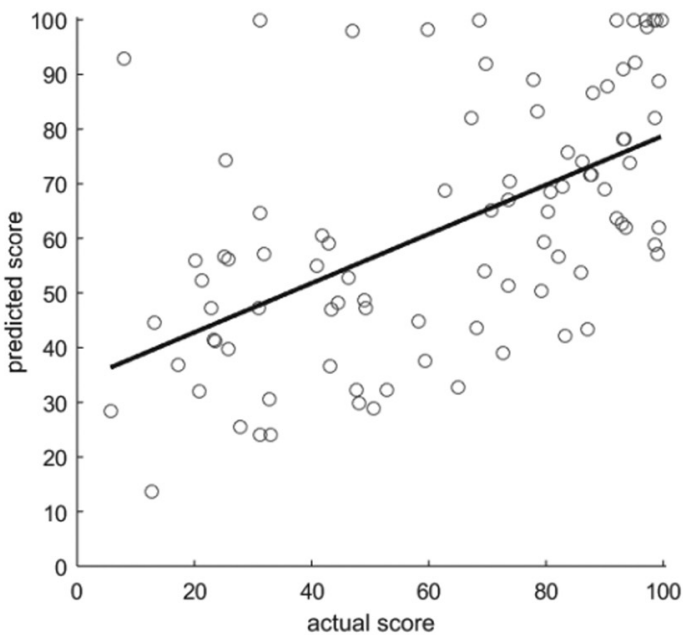

Figure 6. Scatter plots of actual versus predicted AQ scores obtained with lesion-based (left) and connectome-based (right) predictions.

lesion, e.g., in the contralesional hemisphere (Crofts et al., 2011; Gratton et al., 2012).

In the current study, the neural basis of behavioral impairment was explored in two separate analyses. First, we predicted the degree of speech impairment based on the extent of primary ischemic damage to a set of predefined gray-matter regions. Second, we computed the connectome of the post-stroke brain by evaluating the integrity of white-matter tracts and used the con- 

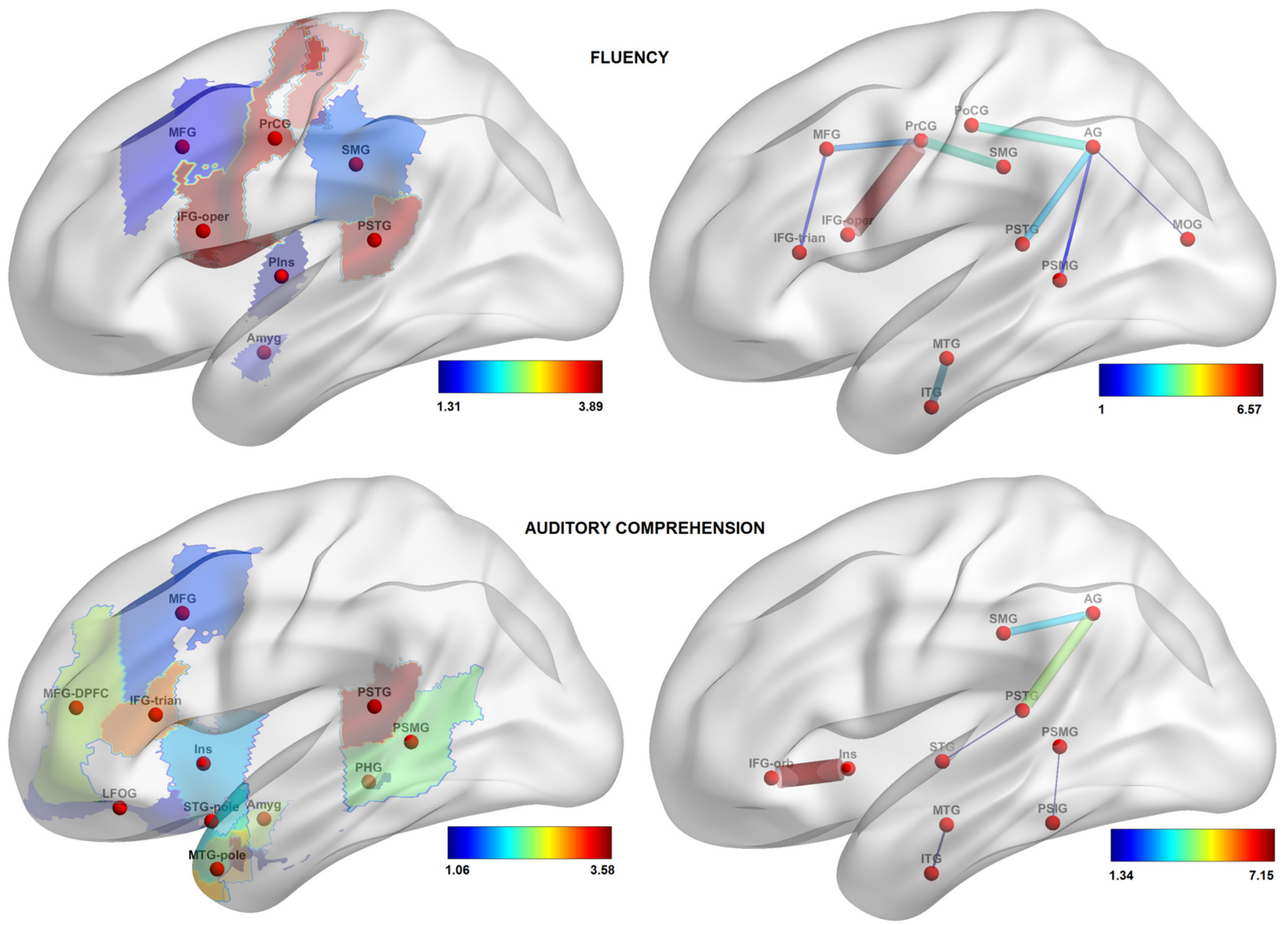

Figure 7. Loadings on the features, reflecting their importance in predicting speech fluency scores (top row) and auditory comprehension scores (bottom row). In the left column, features are gray-matter cortical regions; in the right column, features are white-matter tracts. For abbreviations, see Table 6.

nectome to predict speech impairment. Both types of analysis were approximately equivalent in terms of prediction accuracy, and, furthermore, prediction of behavioral impairment from overall lesion size yielded comparable levels of accuracy. This is expected because damage in some regions highly correlates with lesion size and consistent with previous reports of lesion size being a good predictor of behavioral impairment ( $\mathrm{Wu}$ et al., 2015). However, our region-based multivariate analysis, in addition to predicting behavioral impairment, allowed us to identify the gray-matter regions and (separately) the white-matter tracts in which integrity was important for preserving a particular speech function. In addition, we predicted the same behavioral scores using the combination of lesion-based and connectomebased data as inputs. Prediction from the combination of two modalities was not, in general, more accurate than prediction from one modality; this could be because the number of inputs in a multivariate analysis led to an increase in model complexity (that is, the number of model parameters that need to be estimated using the same amount of training data), which could outweigh the advantage of complementary information provided by the two modalities.

Although the two analyses are complementary and based on data from different neuroimaging modalities (structural MRI and DTI), they are not fully independent, because the brain damage at the lesion locus is likely to involve both gray and white matter. Because the lesion site was masked during probabilistic tractography, it is not surprising that the amount of ischemic damage in the region was correlated with the strength of probabilistic connections stemming from that region (Fig. 3). However, connectome-based analysis provided an additional degree of spatial sensitivity: identification of particular connections in a damaged region that were deemed relevant for a given linguistic function. Furthermore, connectome-based predictions were better than chance even if the connections involving lesioned regions were excluded from the analysis; this suggests that connectome-based analysis can potentially overcome the spatial bias toward areas that have a higher chance of being lesioned because of their position within the vascular bed. For example, the position of insula relative to the middle cerebral artery makes it particularly vulnerable to ischemic stroke; this makes insular regions highly significant predictors of speech impairment in voxelwise symptom mapping analysis (Dronkers, 1996), but this significance could be driven by the vulnerability of insula rather than by its role in speech production (Hillis et al., 2004).

Because of the similarity between post-stroke regional damage and connectome integrity, it is not surprising to see a high degree of correspondence in feature maps computed for the two types of analyses (Figs. 7-9). However, the connectome-based analysis provides some additional information about the cortical networks that support language processing. For example, connectome-based analysis identifies the connections within pa- 

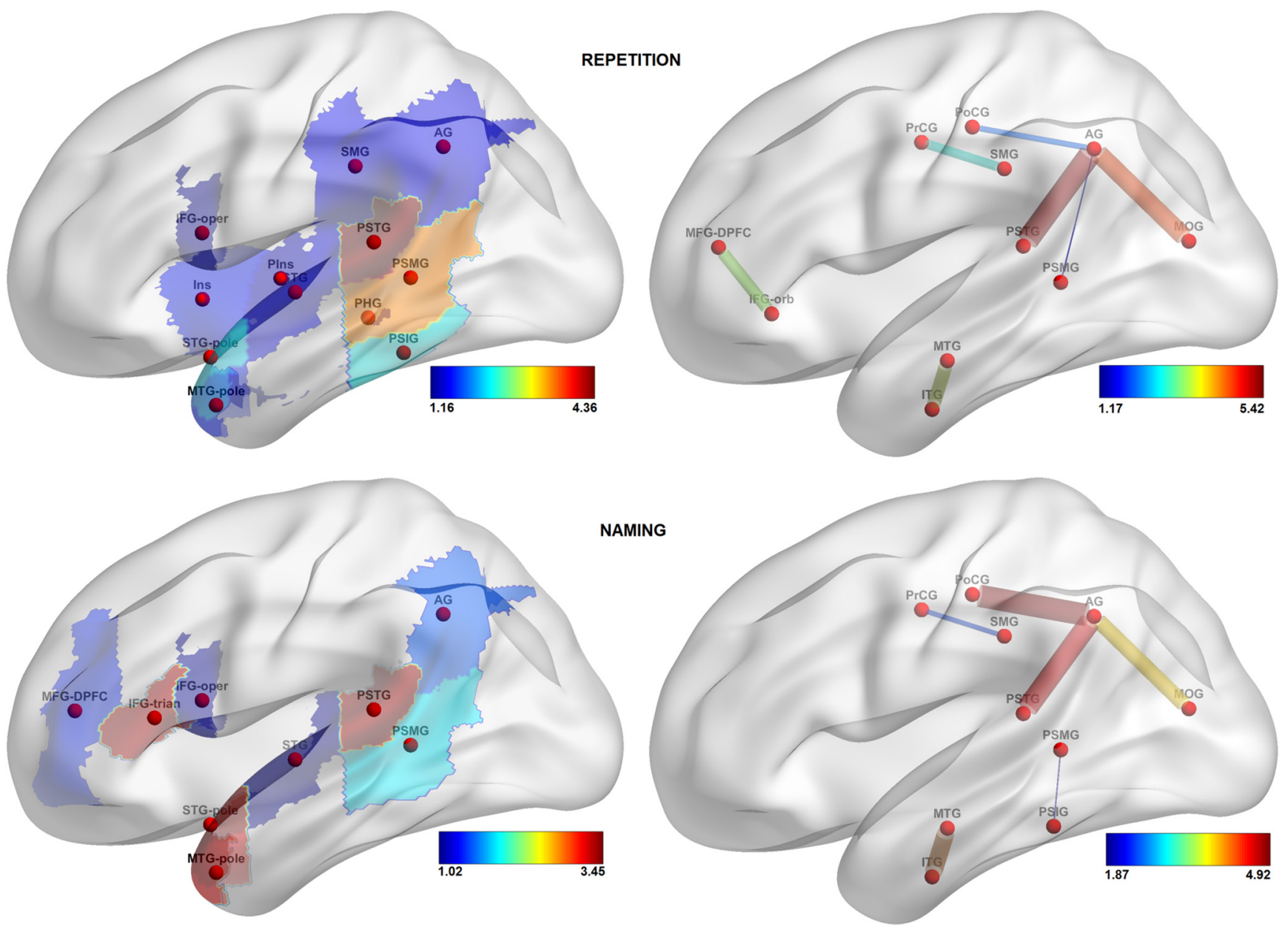

Figure 8. Loadings on the features, reflecting their importance in predicting speech repetition scores (top row) and naming scores (bottom row). In the left column, features are gray-matter cortical regions; in the right column, features are white-matter tracts. For abbreviations, see Table 6.
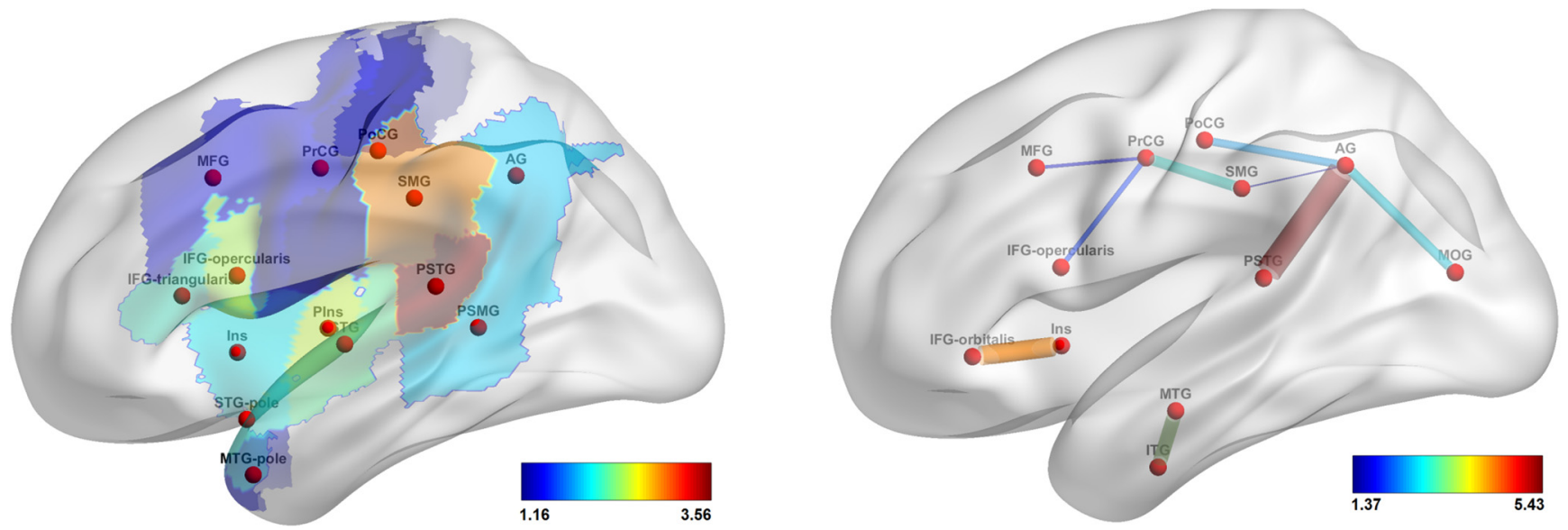

Figure 9. Loadings on the features for AQ prediction (lesion-based, left; connectome-based, right). For abbreviations, see Table 6.

rietal and within orbito-frontal regions as implicated in auditory comprehension and speech repetition, respectively; damage to these regions was not found to be predictive in lesion-based analysis. In addition, there are some regions in which the damage is predictive of language impairment, and connectome-based analysis identifies which particular links stemming from that region are involved. For instance, lesion-based analysis consistently identifies the PSTG as a highly predictive region; from the connectome-based analysis, we know that it is the link from the PSTG to the angular gyrus that is most implicated in the four language modalities that were measured in our study. The PSTG and angular gyrus lie adjacent to each other on the two sides of the junction of the temporal and parietal lobes; this junction has been described as the interface between the motor and the sensory systems, which translates auditory information (e.g., syllables) into motor information (commands to the vocal tract) and vice 
Table 6. Abbreviations of brain regions shown in Figures 7-9

\begin{tabular}{ll}
\hline AG & Angular gyrus \\
Amyg & Amygdala \\
IFG-orb & Inferior frontal gyrus, pars orbitalis \\
IFG-oper & Inferior frontal gyrus, pars opercularis \\
IFG-trian & Inferior frontal gyrus, pars triangularis \\
Ins & Insula \\
ITG & Inferior temporal gyrus \\
LFOG & Lateral fronto-orbital gyrus \\
MFG & Middle frontal gyrus \\
MFG-DPFC & Middle frontal gyrus (dorsal prefrontal \\
& cortex) \\
MOG & Middle occipital gyrus \\
MTG & Middle temporal gyrus \\
MTG-pole & Pole of middle temporal gyrus \\
PHG & Parahippocampal gyrus \\
PIns & Posterior insula \\
POCG & Postcentral gyrus \\
PrCG & Precentral gyrus \\
PSIG & Posterior inferior temporal gyrus \\
PSMG & Posterior middle temporal gyrus \\
PSTG & Posterior superior temporal gyrus \\
SMG & Supramarginal gyrus \\
STG & Superior temporal gyrus \\
STG-pole & Pole of superior temporal gyrus \\
\hline
\end{tabular}

Table 7. Accuracy of prediction of WAB scores from the combination of lesion and connectome data

\begin{tabular}{lll}
\hline WAB score & Prediction accuracy & Number of predictive features \\
\hline Fluency & $0.7458(p=3.34924 \mathrm{e}-17)$ & 18 regions, 15 connections \\
Auditory comprehension & $0.4834(p=1.39251 \mathrm{e}-06)$ & 12 regions, 8 connections \\
Repetition & $0.6189(p=7.96468 \mathrm{e}-11)$ & 17 regions, 7 connections \\
Naming & $0.5843(p=1.49216 \mathrm{e}-09)$ & 14 regions, 11 connections \\
$\mathrm{AQ}$ & $0.6939(p=3.38324 \mathrm{e}-14)$ & 21 regions, 13 connections \\
\hline
\end{tabular}

Table 8. Prediction accuracy of WAB scores on a reduced connectome (consisting of regions that were at least $95 \%$ intact)

\begin{tabular}{lc}
\hline WAB score & \multicolumn{1}{c}{ Prediction accuracy } \\
\hline Speech fluency & $0.3988(p=0.0001)$ \\
Auditory comprehension & $0.3014(p=0.0039)$ \\
Speech repetition & $0.3897(p=0.00015)$ \\
Naming & $0.3664(p=0.0004)$ \\
AQ & $-0.0653(p=0.5407)$ \\
\hline
\end{tabular}

versa (Hickok et al., 2003, 2011). The angular gyrus was found to be more predictive when its connectivity (rather than frank damage) was analyzed. This region is one of the major cortical hubs implicated in a multitude of functions, including speech and language (Seghier, 2013). The connection between angular and middle occipital gyri, which was strongly predictive of speech repetition and naming performance in our study, could be a part of the projections from the angular to the parahippocampal gyri, which can pass through the occipital lobe (Rushworth et al., 2006). Overall, our findings are mostly consistent with previous VLSM (Baldo et al., 2006, 2013; Turken and Dronkers, 2011; Geva et al., 2012; Schwartz, 2014; Weiss et al., 2016) and fMRI (Liljeström et al., 2008; Saur et al., 2008; Meinzer et al., 2009) studies of the neural correlates of fluency, comprehension, repetition, and naming tasks.

This is the first study to assess the whole-brain connectome as a tool to map the relationship between aphasia and white-matter connectivity. Connectomes were constructed using probabilistic tractography; this method, despite its high computational com- plexity, is particularly sensitive in tracking nondominant fiber populations (Behrens et al., 2007). Our predictive analysis was performed on probabilistic fiber count between pairs of regions, which could be a noisy measure of connectivity; an interesting direction for future research is prediction of behavioral impairment from the graph-theoretical measures of binarized (thresholded) connectomes (Rubinov and Sporns, 2010). We opted to include only cortical regions to permit a more equivalent comparison with lesion data, because the inclusion of subcortical links would greatly expand the number of inputs into the multivariate model and potentially decrease its accuracy. Unquestionably, basal nuclei connectivity is important for language and aphasia, and its investigation constitutes an important future use of connectome-based methods. Another possible limitation is our feature-selecting procedure; by discarding the features that were not highly correlated with a given behavioral score, we could potentially discard the features that might be predictive when used in combination with other features. Using alternative methods of feature selection (e.g., based on principal components of the data matrix; Yourganov et al., 2014) is a promising direction for future research.

An additional limitation of our connectome-based analysis is our constraint on the inclusion of white-matter connections. There are 1378 possible connections between 53 left-hemisphere regions, and we retained only 84 for our analysis (only the connections with highly reproducible contralesional homologs were retained). As shown on Figure 2, these connections are mostly between adjacent gray-matter regions. This emphasis on shortrange tracts was a factor in the similarity of the connectomebased predictions to lesion-based predictions. Many important white-matter tracts were not included in our connectome-based analysis because their homologs could not be traced reliably; of particular importance was the omission of the uncinate fasciculus, which is highly important for naming (Papagno, 2011). Likewise, direct links between inferior frontal areas and the areas in the neighborhood of the temporoparietal junction were not included; these links form an important part of the arcuate fasciculus, and it has been known since the days of Karl Wernicke that damage to these connections is implicated in problems with repetition (Geschwind, 1965). Perhaps because of these omissions, the connectome-based analysis was less accurate than lesion-based analysis in predicting the naming and speech repetition scores.

Despite this drawback, the connectome-based analysis was only slightly less accurate than lesion-based analysis in predicting speech fluency, speech repetition, and naming scores and more accurate in predicting auditory comprehension scores. It also identified the important connections between the regions that were missed by lesion-based analysis. In addition, when we ran the connectome-based analysis only on connections between intact regions that were outside of the lesion loci, we could still predict the WAB subscores to some extent (Table 8 ). This shows that the connectome-based analysis uses the information that is not captured by lesion maps; because the spatial occurrence of the lesions is strongly tied to the brain vasculature, the analysis of the connectome can potentially overcome the vasculature-imposed limitations of lesion-based analysis and provide a fuller picture of brain-behavior relationship.

\section{References}

Ashburner J, Friston KJ (2005) Unified segmentation. Neuroimage 26: 839-851. CrossRef Medline

Baldo JV, Schwartz S, Wilkins D, Dronkers NF (2006) Role of frontal versus temporal cortex in verbal fluency as revealed by voxel-based lesion symptom mapping. J Int Neuropsychol Soc 12:896-900. CrossRef Medline Baldo JV, Arévalo A, Patterson JP, Dronkers NF (2013) Grey and white matter 
correlates of picture naming: evidence from a voxel-based lesion analysis of the Boston Naming Test. Cortex 49:658-667. CrossRef Medline

Bates E, Wilson SM, Saygin AP, Dick F, Sereno MI, Knight RT, Dronkers NF (2003) Voxel-based lesion-symptom mapping. Nat Neurosci 6:448-450. CrossRef Medline

Behrens TE, Berg HJ, Jbabdi S, Rushworth MF, Woolrich MW (2007) Probabilistic diffusion tractography with multiple fibre orientations: what can we gain? Neuroimage 34:144-155. CrossRef Medline

Bonilha L, Nesland T, Rorden C, Fillmore P, Ratnayake RP, Fridriksson J (2014) Mapping remote subcortical ramifications of injury after ischemic strokes. Behav Neurol 2014:215380. CrossRef Medline

Bressler SL, Menon V (2010) Large-scale brain networks in cognition: emerging methods and principles. Trends Cogn Sci 14:277-290. CrossRef Medline

Chang CC, Lin CJ (2011) LIBSVM: a library for support vector machines. ACM Trans Interact Intell Syst 2:article 27.

Crofts JJ, Higham DJ, Bosnell R, Jbabdi S, Matthews PM, Behrens TE, Johansen-Berg H (2011) Network analysis detects changes in the contralesional hemisphere following stroke. Neuroimage 54:161-169. CrossRef Medline

Dronkers NF (1996) A new brain region for coordinating speech articulation. Nature 384:159-161. CrossRef Medline

Dronkers NF, Wilkins DP, Van Valin RD Jr, Redfern BB, Jaeger JJ (2004) Lesion analysis of the brain areas involved in language comprehension. Cognition 92:145-177. CrossRef Medline

Faria AV, Joel SE, Zhang Y, Oishi K, van Zjil PC, Miller MI, Pekar JJ, Mori S (2012) Atlas-based analysis of resting-state functional connectivity: Evaluation for reproducibility and multi-modal anatomy-function correlation studies. Neuroimage 61:613-621. CrossRef Medline

Fridriksson J, Bonilha L, Rorden C (2007) Severe Broca's aphasia without Broca's area damage. Behav Neurol 18:237-238. CrossRef Medline

Geschwind N (1965) Disconnexion syndromes in animals and man. I. Brain 88:237-294. CrossRef Medline

Geva S, Baron JC, Jones PS, Price CJ, Warburton EA (2012) A comparison of VLSM and VBM in a cohort of patients with post-stroke aphasia. Neuroimage 1:37-47. CrossRef Medline

Gratton C, Nomura EM, Pérez F, D’Esposito M (2012) Focal brain lesions to critical locations cause widespread disruption of the modular organization of the brain. J Cogn Neurosci 24:1275-1285. CrossRef Medline

Hagmann P, Cammoun L, Gigandet X, Meuli R, Honey CJ, Wedeen VJ, Sporns O (2008) Mapping the structural core of human cerebral cortex. PLoS Biol 6:e159. CrossRef Medline

Henseler I, Regenbrecht F, Obrig H (2014) Lesion correlates of patholinguistic profiles in chronic aphasia: comparisons of syndrome-, modality-, and symptom-level assessment. Brain 137:918-930. CrossRef Medline

Hickok G, Poeppel D (2007) The cortical organization of speech processing. Nat Rev Neurosci 8:393-402. CrossRef Medline

Hickok G, Buchsbaum B, Humphries C, Muftuler T (2003) Auditory-motor interaction revealed by fMRI: speech, music, and working memory in area Spt. J Cogn Neurosci 15:673-682. CrossRef Medline

Hickok G, Houde J, Rong F (2011) Sensorimotor integration in speech processing: computational basis and neural organization. Neuron 69:407422. CrossRef Medline

Hillis AE, Work M, Barker PB, Jacobs MA, Breese EL, Maurer K (2004) Re-examining the brain regions crucial for orchestrating speech articulation. Brain 127:1479-1487. CrossRef Medline

Kertesz A (1982) Western aphasia battery test manual. New York: Psychological Corp.

Liljeström M, Tarkiainen A, Parviainen T, Kujala J, Numminen J, Hiltunen J, Laine M, Salmelin R (2008) Perceiving and naming actions and objects. Neuroimage 41:1132-1141. CrossRef Medline

Luria AR (1966) Higher cortical functions in man. New York: Basic Books.

Mah YH, Husain M, Rees G, Nachev P (2014) Human brain lesion-deficit inference remapped. Brain 137:2522-2531. CrossRef Medline

McIntosh AR (1999) Mapping cognition to the brain through neural interactions. Memory 7:523-548. CrossRef Medline

McIntosh AR (2000) Towards a network theory of cognition. Neural Networks 13:861-870. CrossRef Medline

Meinzer M, Flaisch T, Wilser L, Eulitz C, Rockstroh B, Conway T, Gonzalez-Rothi L, Crosson B (2009) Neural signatures of semantic and phonemic fluency in young and old adults. J Cogn Neurosci 21:2007-2018. CrossRef Medline

Mesulam MM (1990) Large scale neurocognitive networks and distributed processing for attention, language, and memory. Ann Neurol 28:597613. CrossRef Medline

Misaki M, Kim Y, Bandettini PA, Kriegeskorte N (2010) Comparison of multivariate classifiers and response normalizations for pattern-information fMRI. Neuroimage 53:103-118. CrossRef Medline

Nachev P, Coulthard E, Jäger HR, Kennard C, Husain M (2008) Enantiomorphic normalization of focally lesioned brains. Neuroimage 39:1215-1226. CrossRef Medline

Papagno C (2011) Naming and the role of the uncinate fasciculus in language function. Curr Neurol Neurosci Rep 11:553-559. CrossRef Medline

Park HJ, Friston K (2013) Structural and functional brain networks: from connections to cognition. Science 342:1238411. CrossRef Medline

Rubinov M, Sporns O (2010) Complex network measures of brain connectivity: uses and interpretations. Neuroimage 52:1059-1069. CrossRef Medline

Rushworth MF, Behrens TE, Johansen-Berg H (2006) Connection patterns distinguish 3 regions of human parietal cortex. Cereb Cortex 16:14181430. CrossRef Medline

Saur D, Kreher BW, Schnell S, Kümmerer D, Kellmeyer P, Vry MS, Umarova R, Musso M, Glauche V, Abel S, Huber W, Rijntjes M, Hennig J, Weiller C (2008) Ventral and dorsal pathways for language. Proc Natl Acad Sci U S A 105:18035-18040. CrossRef Medline

Schmah T, Yourganov G, Zemel RS, Hinton GE, Small SL, Strother SC (2010) Comparing classification methods for longitudinal fMRI studies. Neural Comput 22:2729-2762. CrossRef Medline

Schwartz MF (2014) Theoretical analysis of word production deficits in adult aphasia. Philos Trans R Soc Lond B Biol Sci 369:20120390. CrossRef Medline

Seghier ML (2013) The angular gyrus multiple functions and multiple subdivisions. Neuroscientist 19:43-61. CrossRef Medline

Smith DV, Clithero JA, Rorden C, Karnath HO (2013) Decoding the anatomical network of spatial attention. Proc Natl Acad Sci U S A 110:1518 1523. CrossRef Medline

Smola AJ, Schölkopf B (2004) A tutorial on support vector regression. Stat Comput 14:199-222. CrossRef

Sporns O (2013) The human connectome: origins and challenges. Neuroimage 80:53-61. CrossRef Medline

Sporns O (2014) Contributions and challenges for network models in cognitive neuroscience. Nat Neurosci 17:652-660. CrossRef Medline

Thomalla G, Glauche V, Weiller C, Röther J (2005) Time course of wallerian degeneration after ischaemic stroke revealed by diffusion tensor imaging. J Neurol Neurosurg Psychiatry 76:266-268. CrossRef Medline

Tononi G (2004) An information integration theory of consciousness. BMC Neurosci 5:42. CrossRef Medline

Turken AU, Dronkers NF (2011) The neural architecture of the language comprehension network: converging evidence from lesion and connectivity analyses. Front Syst Neurosci 5:1. CrossRef Medline

Weiss PH, Ubben SD, Kaesberg S, Kalbe E, Kessler J, Liebig T, Fink GR (2016) Where language meets meaningful action: a combined behavior and lesion analysis of aphasia and apraxia. Brain Struct Funct 221:563576. CrossRef Medline

Wu O, Cloonan L, Mocking SJ, Bouts MJ, Copen WA, Cougo-Pinto PT, Fitzpatrick K, Kanakis A, Schaefer PW, Rosand J, Furie KL, Rost NS (2015) Role of acute lesion topography in initial ischemic stroke severity and long-term functional outcomes. Stroke 46:2438-2444. CrossRef Medline

Xia M, Wang J, He Y (2013) BrainNet viewer: a network visualization tool for human brain connectomics. PLoS One 8:e68910. CrossRef Medline

Yourganov G, Schmah T, Churchill NW, Berman MG, Grady CL, Strother SC (2014) Pattern classification of fMRI data: applications for analysis of spatially distributed cortical networks. Neuroimage 96:117132. CrossRef Medline

Yourganov G, Smith KG, Fridriksson J, Rorden C (2015) Predicting aphasia type from brain damage measured with structural MRI. Cortex 73:203215. CrossRef Medline

Yuste R (2015) From the neuron doctrine to neural networks. Nat Rev Neurosci 16:487-497. CrossRef Medline

Zeki S, Watson JD, Lueck CJ, Friston KJ, Kennard C, Frackowiak RS (1991) A direct demonstration of functional specialization in human visual cortex. J Neurosci 11:641-649. Medline

Zhang Y, Kimberg DY, Coslett HB, Schwartz MF, Wang Z (2014) Multivariate lesion-symptom mapping using support vector regression. Hum Brain Mapp 35:5861-5876. CrossRef Medline 